\section{Laser spectroscopy of}

\section{nanometric gas cells}

Martial Ducloy

Laboratoire de Physique des Lasers, Université Paris Nord, Villetaneuse, France

7 he Doppler effect was predicted by Christian Doppler during 1 his stay in Prague in 1842 [1]. This effect is related to the propagation of oscillating waves (e.g., sound waves, or light waves) and predicts the change of frequency of a wave emitted by a source, which is not static and moves either in the direction of the observer, or away from it : when the source approaches the observer, there is an increase in the frequency of the wave detected by the observer ("blue shift"), and when the source recedes, there is a decrease in the frequency ("red shift").

The Doppler effect plays an important role in the optical spectra of atomic or molecular species, from which one can analyse their characteristic emission frequencies. When these elements are in the gas phase (vapours, discharges, etc.), they are moving at random velocities, the distribution of which is centred at an average velocity $u$ governed by the gas temperature (indeed proportional to its square root). This distribution of velocities induces a frequency broadening of the atomic emission lines (thermal or "Doppler" line-width), proportional to $v_{a t} u / c$ ( $v_{a t}$, atomic frequency; $c$, light velocity). The emission frequencies, which are essential for our understanding of the internal dynamics of atoms or molecules, and of their fundamental properties, cannot be measured with an accuracy better than the Doppler broadening.

The laser era, starting in 1960, revolutionised the field of optical spectroscopy. With the advent of laser sources, which are sources of monochromatic optical light that have a very high spectral purity (e.g., their oscillation frequency $v$ has an extremely precise definition-in some cases, better than $10^{15}$ ), and a very large brilliance or intensity, optical absorption spectroscopy took on a great importance. In particular, non-linear absorption spectroscopy became a very useful tool [2].

Among these methods, saturated absorption spectroscopy appeared as a method of paramount importance to get rid of the

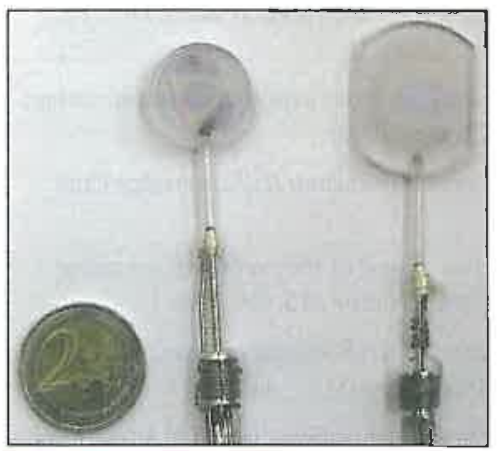
Doppler broadening of spectral lines. It makes use of the non-linear response of a resonant atomic or molecular gas to the irradiation by two counter-propagating laser waves of the same frequency $v_{0}$. One wave excites the atomic species for a given velocity $\mathrm{v}_{\mathrm{z}}$, determined by the reso-

Fig. 1: Photograph of "pill-box" cells. The interferometric modulation (fringes) of light reflected from the input cell of the window can be seen on the pictures. Each fringe corresponds to a thickness change of $\lambda / 2$. The coin of 2 Euros (diameter $25 \mathrm{~mm}$ ) gives the scale of the figure.

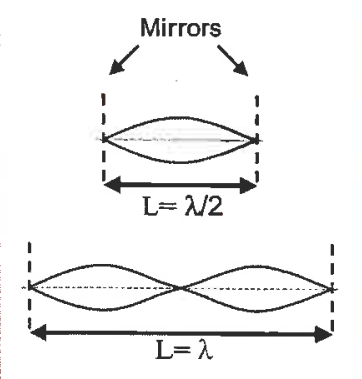

4ig. 2: Vibration mode of a string fixed in two points at a distance $L$. The string oscillation is maximum ("resonance") for $\mathrm{L}=$ $\lambda / 2$ (fundamental note, one spatial maximum), $L=\lambda$ (harmonics 2 , two spatial maximums), $L=3 \lambda / 2$, etc. An electromagnetic field propagating back and forth between two parallel mirrors (dotted lines) facing each other, reproduces the same type of resonant behaviour, because of the cancellation of the electric field at the mirrors' position.

nance condition $v=v_{a t}$, where $v$ is the light frequency, taking into account the Doppler shift, $v=v_{0}\left(1-\mathrm{v}_{z} / \mathrm{c}\right)$. Because the other wave is counter-propagating to the first one, it probes atoms with opposite velocity, $-v_{\mathrm{z}}$. Therefore, only for $\mathrm{v}_{\mathrm{z}}=0$, the first wave will modify the propagation characteristics (absorption, dispersion, etc.) of the opposite wave, because of their simultaneous interaction with the same group of atoms having a zero velocity component along the propagation axis. This happens only for a laser exactly tuned to the atomic frequency $v_{0}=v_{a t}$, and allows for the elimination of the Doppler broadening, because of the velocity selection by a monochromatic laser source [2].

An original approach, which has been pioneered by the late Venyamin Chebotaev of the Institute of Laser Physics, relies on the simultaneous absorption of quanta of light ("photons") coming from laser beams propagating in well-defined directions inside the atomic gas. In particular, if the atom absorbs simultaneously two photons of the same frequency, each travelling in counter-propagating directions, the Doppler shifts respectively associated with either absorption are exactly opposite, and their sum cancels, leading to a zero Doppler shift, whatever the atomic velocity may be [3]. This powerful technique, called "Doppler-free two-photon absorption" has been successfully applied to the spectral analysis of many atomic or molecular species.

More recently, another approach, developed in the 1980's, has relied on stopping and trapping atoms by using the radiation pressure induced on atoms by resonantly absorbed light [4]. When one photon is absorbed by an atom, this atom undergoes a kick ("photon recoil") in the direction opposite to the propagation direction of the photon. Atoms absorbing a photon propagating in a direction opposite to their motion are then slightly decelerated because of this recoil kick. They are finally stopped when they have absorbed enough photons. This is the very rapidly developing field of control of atomic motion by laser light [4]. For these stationary atoms, the Doppler shift is cancelled.

In the following a different approach is presented which makes use of ultra thin cells of atomic gas as laser light absorbers.

\section{Gas dynamics and light absorption in ultra thin gas cells}

"Ultra thin cells" are gas cells in which one dimension of the cell is much smaller than the other ones. They can be called "pill-box" cells [5]. Examples of such cells are given in Fig.1. We have used such cells with thickness, $L$, which can vary from 10-20 $\mu \mathrm{m}$, for the most common ones, down to $1 \mu \mathrm{m}$ or even $100 \mathrm{~nm}$, for the most recent ones [6]. The cell transverse dimensions are of the order of $2 \mathrm{~cm}$. The thinnest cells present an aspect ratio of 200000 : to give an idea, this would correspond to digging a 60 metre-wide hole, throughout the earth, between the two poles, or equivalently, to a 
corridor, of width 1.8 $\mathrm{km}$, linking the earth to the moon. The fabrication of these cells needs a special technology that has been described elsewhere (see Ref. [6]).

An important point is to know the cell thickness $\mathrm{L}$ accurately. To measure it, one makes use of the fact that the windows of the cell are partially reflecting the light (Fig.1). Thanks to the presence of two-

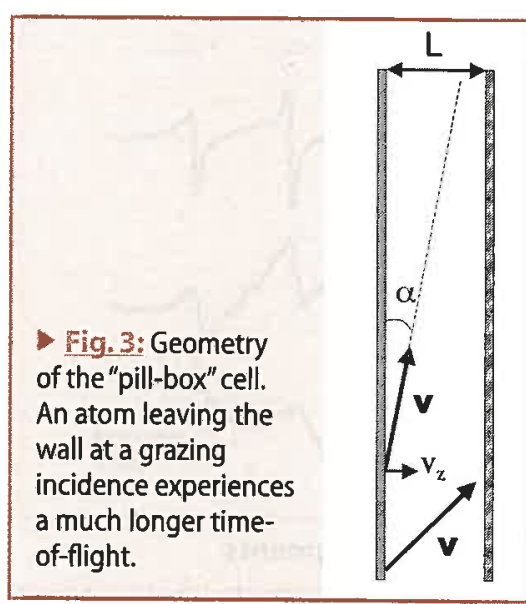

input/output - windows, light reflection on the pill-box cell exhibits an interferometric behaviour as a function of the cell thickness. This is the so-called "Fabry-Perrot" effect that is related to the oscillating wave properties of light. One can understand it by analogy with the vibrations of a fiddle string: the oscillation amplitude of the string is enhanced when its length is equal to half of the oscillation wavelength (fundamental note) or to a multiple of this half wavelength ("harmonics") (Fig.2). These resonances correspond to a zero motion of the two extremities of the string which are fixed on a support. Similarly, a set of two parallel mirrors behave like a resonator for light-the "Fabry-Pérot" resonator: analogous resonances appear in the amplitude of light oscillating between the two mirrors (at the mirror position, the electric field must cancel). When the mirror spacing is a multiple of half the light wavelength $\lambda$, the transmission of incident light is a maximum, at the same time that its reflection is a minimum. The same behaviour applies to light reflection on a "pill-box" cell, with reflection minima occurring each time its thickness $\mathrm{L}$ is equal to $n$ times $\lambda / 2$. By monitoring light reflection for several wavelengths, one can then determine $L$ with a very good accuracy (better than $4 \mathrm{~nm}$ ) in each point of the cell.

What is the dynamics of atoms inside this very peculiar type of "pillbox" cell? As an example, one can consider Caesium (Cs) atoms at room temperature: their mean thermal velocity is $200 \mathrm{~m} / \mathrm{s}$. Then for a $1 \mu \mathrm{m}$-thick cell, an atom flies, from one wall to the other, in an average time $t=5 \mathrm{~ns}$. By comparison the residence time of Cs atoms on a silica wall ("adsorption" time) is on the order of $5 \mu \mathrm{s}$ : there are one thousand more atoms adsorbed on the walls than freely-moving atoms in the gas!

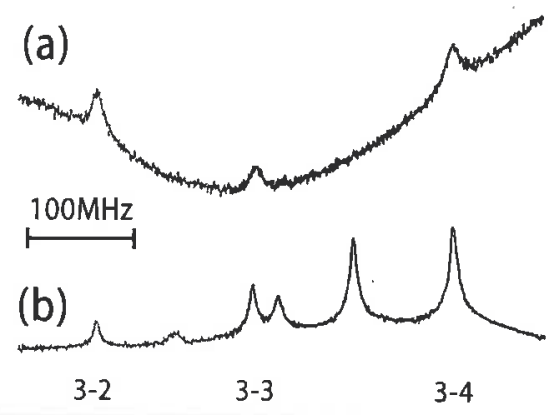

Aig. 4: (a) Light transmission spectrum of a $10 \mu \mathrm{m}$-thick Cs cell, monitored near the $C s D_{2}$ resonance line at $\lambda=852 \mathrm{~nm}$. The narrow spectral components are characteristic of the cell geometry and free of Doppler effect. They are revealing the atomic hyperfine structure of Cs. (b) Reference spectrum obtained by "saturated absorption" in a large cell (from Ref. [7]).
A second noteworthy property of the gas dynamics is the fact that the time of flight of an atom between the two walls is highly anisotropic (Fig.3). An atom moving normal to the surfaces has an average "lifetime" of about 5 ns, while an atom leaving the surface at grazing incidence has a much longer time of flight. For example, for a grazing angle $\alpha$ of one degree between the atom's velocity and the plane of the surfaces, the time of flight increases to about $0.3 \mu \mathrm{s}$. The dramatic consequence is that the atoms that are moving nearly parallel to the surface interact for a much longer time with the light, and thereby contribute dominantly to the light absorption. When the incident light is normal to the surface, the Doppler effect cancels for those atoms moving at grazing incidence: the light absorption becomes free of Doppler broadening, as has been demonstrated in thin cells of Cs gas at the $\mathrm{D}_{2}$ resonance line, $\lambda=852 \mathrm{~nm}$ (Fig. 4 , from Ref. [7]).

\section{Singular properties of light transmission in ultra thin gas cells}

The absorption spectrum shown in Fig. 4 has been recorded on a gas cell of thickness $\mathrm{L}=10 \mu \mathrm{m}$. When $\mathrm{L}$ is decreased to reach values of the order of the wavelength $\lambda$, novel coherent effects appear.

\section{Fabry-Pérot effects}

First, because of the "Fabry-Pérot" character of the two highly parallel windows (see previous section and Fig.2), the light amplitude is spatially modulated inside the cell, with intensity maxima when $\mathrm{L}=n \lambda / 2$ ( $n$, integer). This intensity modulation strongly alters the overall amplitude of absorption spectra when $\mathrm{L}$ is varied, as well as that of reflection spectra [8].

\section{Coherent character of the transient atomic response}

The response of atoms to laser light excitation is essentially a transient one. Indeed the atoms adsorbed on one of the walls are perturbed in such a dramatic way that they cannot interact with the light. They are in equilibrium, i.e., when they leave the wall, their internal energy is minimum and they are in the "ground state". Then, as soon as an atom quits the surface, it starts interacting with the resonant incident laser light and enters a transient absorption process. This transient response, which lasts for the whole time-of-flight of the atom in the case of ultra thin cells, is characterised by an oscillation of the atom's electronic charges (electric

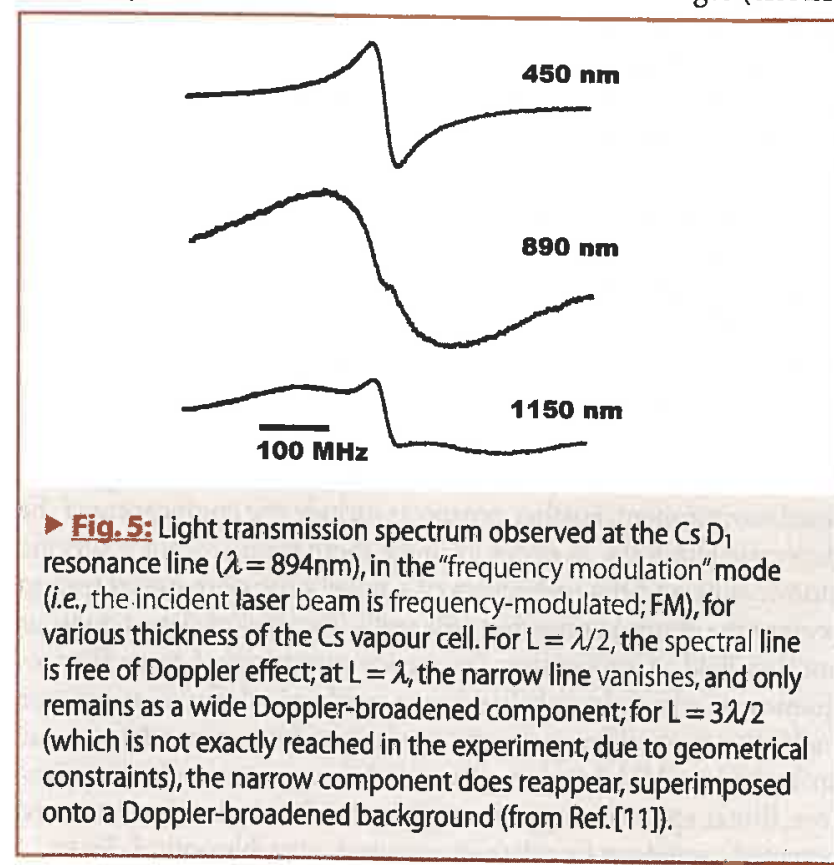


dipole moment of the atom) driven by the oscillating electric field of the light. The phase of oscillation of the atomic dipole moment depends on its position in the cell. Summing up the various atomic dipole contributions to light absorption depends on their relative phase and leads to constructive or destructive interference, according to the cell length $[9,10]$. One has predicted and experimentally observed (Fig. 5) that the "Doppler-free" absorption resonance reaches a maximum amplitude for $L=\lambda / 2,3 \lambda / 2$, etc., and cancels for $\mathrm{L}=\lambda, 2 \lambda \ldots[11]$. This length dependence is a clear signature of the coherent character of light absorption in ultra thin gas cells.

\section{Wall attraction exerted on atoms}

For still thinner cells ( $\mathrm{L}<200 \mathrm{~nm})$, the atoms start to feel the presence of the cell walls, even when they are moving at the centre of the cell. There is an attractive force between the atom and the surfaces. The electronic charges of the atom polarise the wall, inducing fictitious charges of opposite sign (the "image" charges) inside it. The resulting attraction between the atom's electronic cloud and its image appears at intermediate distances, $d \cong 10-100 \mathrm{~nm}$; this is the so-called long-range, "van der Waals," or "London - van der Waals," atom-surface interaction [12].

Such a surface interaction acts on the atom as an external attractive potential, which lowers its internal energy. This attraction also shifts the resonance lines of atoms located close to a surface [13], because its strength depends on the atom internal state: for excited states of the atom, the electronic cloud is larger than for the ground state, implying larger charge fluctuations and larger surface attraction. Then, since the excited state energy is lowered more than that of the ground state, the atomic resonance frequency becomes smaller: this is the wall-induced "red shift" of the atomic resonance, which increases with decreasing atom-surface distances. This red shift has been observed in the transmission spectra of ultra-thin gas cells (Fig.6), and its thickness dependence (increased frequency shift with decreasing thickness) has been checked [14]. This is direct evidence of the ubiquitous van der Waals forces between neutral polarisable systems (here, an atom and a surface) which are of paramount importance in many fields of science and technology: physics, chemistry, biology, nanotechnologies...

\section{Conclusion}

Spectroscopy of microscopic (nanoscopic) gas cells gives access to a new regime of gas dynamics in which the thermodynamical equilibrium bscomes cell-specific. The corresponding atom-light interaction exhibits peculiar features, including a fully transient and coherent response, a strongly anisotropic atom time-of-flight and an explored space smaller than the wavelength. Transmission spectroscopy of ultra-thin gas cells allows one to study the field of Quantum Electrodynamics inside a dielectric nanocavity (where light is confined in a sub-wavelength space), as well as nanophysics and nanotechnology with "quasi interaction-free" atoms. It offers a way to explore longrange atom-surface interactions in a range of distances $(\sim 10 \mathrm{~nm})$ which has not been studied before. It should also give access to the peculiar characteristics of atom-atom interactions in a strongly confined environment. Further prospects include the engineering of the dielectric windows, in order to make them repulsive for a specific atomic state and the realisation of a novel type of material trap to prevent the atoms approaching the walls. To conclude let us quote as another field of application, frequency metrology. A laser, the frequency of which is stabilised on a perfectly defined atomic or molecular spectral line, provides an absolute frequency reference, i.e. an "optical clock". Ultra thin cells, with their opportunity for Dopplerfree, linear spectroscopy in a tightly confined environment, are potential candidates for relatively compact, portable optical clocks.

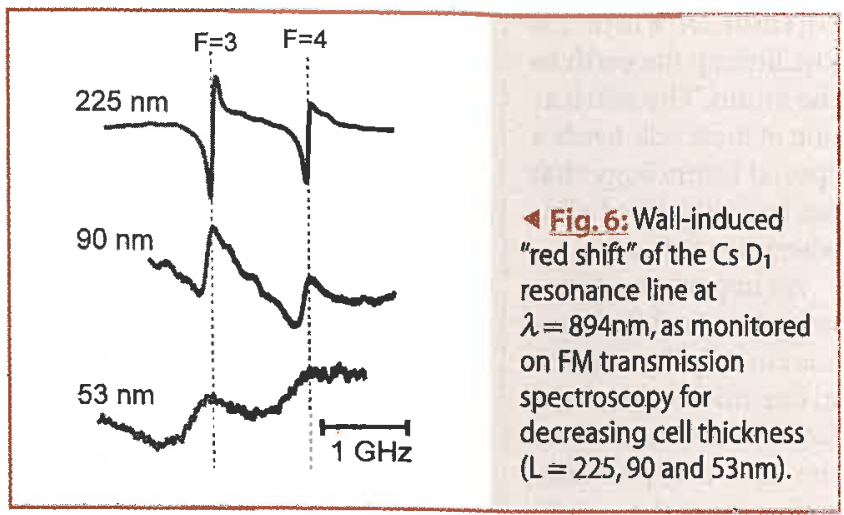

\section{Acknowledgments}

This article is adapted from a presentation given at the general assembly of the Russian Academy of Sciences (RAS, Siberian Section, Novosibirsk) when the author received the honorary degree of foreign member of the RAS. The experiments presented here have been performed in "Laboratoire de Physique des Lasers" (LPI, Université Paris Nord), in collaboration with the Institute for Physical Research (IPR, Ashtarak, Armenia), where some of the technology of ultra thin cells has been developed. The author would like to thank Daniel Bloch, Stephan Briaudeau, Gabriel Dutier, Michèle Fichet and Marie-Pascale Gorza (LPL), David Sarkysian and Aram Papoyan (IPR), Alexander Yarovitski (Lebedev Physical Institute, Moscow) and Solomon Saltiel (Sofia University, Bulgaria) for their major contributions to this work.

\section{About the author}

Martial Ducloy graduated from Ecole Normale Supérieure and got his "Doctorat d'Etat" from Paris University in 1973. He is presently "Directeur de Recherche" CNRS, at Université Paris 13 (Villetaneuse), where he has been head of the Laser Physics Laboratory, Vice-President for Research (1986-93) and Dean for International Relations (1996-2002). He is Past-President of the EPS, and former chair of the EPS Quantum Electronics and Optics Division.

\section{References}

[1] See, e.g., Christian Doppler's biography in "EPS Biographies of European Physicists", Vol.1 (European Physical Society, Mulhouse, France, 2003)

[2] See, e.g., V. S. Letokhov and V. P. Chebotaev, "Nonlinear Laser Spectroscopy" (Springer Verlag, Berlin, 1977)

[3] L. S. Vasilenko, V. P. Chebotaev and A. V. Shishaev, Pis'ma Zh. Eksp. Teor. Fiz. 12, 161 (1970) [JETP Letters 12, 113 (1970)]; B. Cagnac, G. Grynberg and F. Biraben, J. Physique (Paris) 34, 845 (1973)

[4] S. Chu, C. Cohen-Tannoudji and W. D. Phillips, 1997 Nobel Lectures, Reviews of Modern Physics 70, 685-741 (1998)

[5] R.H. Romer and R.H. Dicke, Physical Review 99, 532 (1955)

[6] D. Sarkisyan et al., Optics Comm. 200, 201 (2001)

[7] S. Briaudeau, D. Bloch and M. Ducloy, Europhysics Letters 35, 337 (1996)

[8] G.Dutier et al., Journal of the Optical Society of America B 20,793 (2003)

[9] B. Zambon and G. Nienhuis, Optics Comm. 143, 308 (1997)

[10] S. Briaudeau et al., Physical Review A 57, R3169 (1998)

[11] G. Dutier et al., Europhysics Letters 63, 35 (2003)

[12] J.E. Lennard-Jones, Transactions of the Faraday Society 28, 334 (1932)

[13] M. Oria et al., Europhysics Letters 14, 527 (1991)

[14] G. Dutier et al., Journal de Physique IV (France) 12, Pr5-155 (2002) 